\title{
La sociolinguistique russe actuelle : deux approches divergentes et non conflictuelles?
}

\section{Margarita Schoenenberger}

\section{(2) OpenEdition}

\section{Journals}

Édition électronique

URL : http://journals.openedition.org/edl/317

DOI : $10.4000 /$ edl. 317

ISSN : 2296-5084

Éditeur

Université de Lausanne

\section{Édition imprimée}

Date de publication : 15 décembre 2009

Pagination : 115-138

ISBN : 978-2-940331-21-5

ISSN : 0014-2026

\section{Référence électronique}

Margarita Schoenenberger, «La sociolinguistique russe actuelle : deux approches divergentes et non conflictuelles? ", Études de lettres [En ligne], 4 | 2009, mis en ligne le 15 décembre 2012, consulté le 18 décembre 2020. URL : http://journals.openedition.org/edl/317; DOI : https://doi.org/10.4000/edl.317 


\section{LA SOCIOLINGUISTIQUE RUSSE ACTUELLE: DEUX APPROCHES DIVERGENTES ET NON CONFLICTUELLES?}

Dans les années 2000, apparaissent à quelques années d'intervalle deux manuels russes de sociolinguistique destinés à l'enseignement supérieur: La sociolinguistique [Sociolingvistika] de L.P. Krysin et V.P. Belikov et La sociolinguistique et la sociologie du langage [Sociolingvistika i sociologija jazyka] de N.B. Vaxtin et E.B. Golovko. Le premier livre s'inscrit dans une tradition russo-soviétique, tandis que les auteurs du second se tournent ouvertement vers la sociolinguistique anglo-américaine pour élaborer leur ouvrage. Il en ressort deux visions assez différentes du fonctionnement social du langage et de ses normes.

\section{Introduction}

Pour les linguistes formés en Occident, c'est aux Etats-Unis que la science du langage connaît, au début des années 1970, un développement rapide, qui a reçu le nom de sociolinguistique et qui réunit dans son objet (l'activité langagière) le social et le linguistique. C'est une science inductive par essence: c'est à partir d'enquêtes de terrain que les chercheurs américains D. Hymes, Ch. Ferguson, J. Gumperz, J. Fishman, W. Labov et d'autres ont élaboré leurs concepts théoriques pour aller de nouveau vers des corpus de faits langagiers réellement produits. Le caractère empirique de la sociolinguistique américaine ne l'a pas empêchée d'élaborer 
des concepts théoriques dès les premiers travaux sociolinguistiques des années 1950-1960 ${ }^{1}$.

La sociolinguistique américaine a connu un succès certain en Europe occidentale, en tout cas on ne pouvait pas l'ignorer. C'était également le cas en Russie. Une sociolinguistique soviétique s'est affirmée à la même époque, représentée surtout par les travaux des linguistes moscovites L.P. Krysin et E.A. Zemskaja. Cette sociolinguistique était étroitement liée à une autre discipline linguistique soviétique à visée sociale, appelée "culture de la langue». Il s'agissait d'une science normative et prescriptive: les linguistes partaient à la recherche de la norme pour justifier leurs prescriptions normatives ${ }^{2}$. Il reste néanmoins qu'aussi bien les linguistes soviétiques que les sociolinguistes occidentaux et américains cherchaient à faire entrer la variation linguistique (norme = choix = variantes) dans leurs descriptions et à expliciter ses causes par des influences externes à la langue. Sont-ils tous pour autant des sociolinguistes ou, autrement dit, appartiennent-ils au même courant scientifique?

Dans les années 2000, apparaissent à quelques années d'intervalle deux manuels russes de sociolinguistique destinés à l'enseignement supérieur ${ }^{3}$. Plus précisément, il sont deux: La sociolinguistique [Sociolingvistika] de V.P. Belikov et L.P. Krysin (2001) et La sociolinguistique et la sociologie du langage [Sociolingvistika $i$ sociologija jazyka] de N.B. Vaxtin et E.B. Golovko (2004). Le premier est édité à Moscou, le second à Saint-Pétersbourg. Les différences ne se résument pourtant pas quau lieu de parution de ces ouvrages. Le livre de Belikov et Krysin s'inscrit, à mon avis, dans une tradition russo-soviétique, même si les auteurs démontrent une connaissance certaine de la sociolinguistique occidentale. Vaxtin et Golovko se tournent ouvertement vers la sociolinguistique anglo-américaine pour élaborer leur ouvrage sans pour autant oublier les linguistes russes dans leur bibliographie. Il en ressort deux

I. Cf. les exemples d'un tel cheminement à partir d'un corpus de données vers l'élaboration de concepts sociolinguistiques dans les travaux de Ch.A. Ferguson pour la notion de "diglossie» (cf. Ch.A. Ferguson, "Diglossia»), de J. Gumperz pour celles de «communauté linguistique» et «loyauté linguistique» (cf. J.J. Gumperz, "The Speech Community»).

2. Pour une analyse détaillée de l'aspect normatif et prescriptif de la sociolinguistique soviétique, cf. M. Schoenenberger, "Une sociolinguistique prescriptive».

3. Le manuel précédent avait été publié en 1978 par A.D. Švejcer et L.B. Nikol'skij (A.D. Švejcer, L.B. Nikol'skij, Vvedenie v sociolingvistiku). 
visions assez différentes du fonctionnement social du langage, de ses normes. Une mise en lumière croisée de ces deux approches permet de discerner des voies fort divergentes que peuvent prendre les sciences du langage en Russie d'aujourd'hui.

J'essayerai de comparer ces deux ouvrages d'après les critères (ou les thèmes) suivants: la place des sources scientifiques russes, la place des sources scientifiques occidentales, la notion de "norme linguistique", la définition et la différenciation des facteurs sociaux dans le fonctionnement linguistique, les notions de «langue standard», «normée» ou «littéraire» et de "variétés non standards».

\section{Place de sources russes}

Aussi bien Krysin et Belikov que Vaxtin et Golovko citent plusieurs auteurs russes parmi les linguistes qui ont mis au centre de leurs recherches le rôle des conditions sociales dans le fonctionnement du langage.

Pour Krysin et Belikov, la sociolinguistique russe a ses propres racines, plus anciennes que la sociolinguistique américaine ("Les chercheurs américains redécouvrent souvent ce qui a déjà été relevé par leurs prédécesseurs européens ${ }^{4}$ ) ou l'étude sociale du langage en France, représentée, pour les auteurs, en premier lieu par A. Meillet et plus tard par A. Martinet. C'est le linguiste russe d'origine polonaise I.A. Baudouin de Courtenay (1845-1929) qui est pour les auteurs de La sociolinguistique le précurseur de la sociolinguistique contemporaine. Le livre de Krysin et Belikov fait une part belle aux linguistes russes qui ont succédé à Baudouin, qui "ont suivi» en quelques sortes ses traces. On y trouve de fréquentes références à E.D. Polivanov, A.M. Seliščev, R.O. Šor, L.P. Jakubinskij, B.A. Larin, V.M. Žirmunskij, M.A. Peterson, V.V. Vinogradov, G.O. Vinokur. La sociolinguistique russe actuelle reconnaît s'appuyer également sur les apports de l'Ecole de Prague, surtout pour l'étude du fonctionnement des langues "littéraires" 5 .

Louvrage de Vaxtin et Golovko s'appuie ouvertement dès les premières pages sur la tradition anglophone dans l'étude sociale du langage. Avec

4. L.P. Krysin, V.I. Belikov, Sociolingvistika, p. 15.

5. Cf. M. Schoenenberger, "Une sociolinguistique prescriptive». 
une liberté de ton qui leur est propre, les auteurs appellent de leurs vœux des investigations sociolinguistiques sur le terrain russe, en affirmant par là même l'absence de telles recherches. Mais on ne trouve pas dans $\mathrm{La}$ sociolinguistique et la sociologie du langage de jugement explicite au sujet de la sociolinguistique russo-soviétique, même si la partie russe de la bibliographie est très bien fournie (le manuel de Krysin et Belikov en fait partie, ainsi que d'autres travaux de ces auteurs). Cependant, Baudouin de Courtenay et Vinogradov, figures de proue de la sociolinguistique russe dans le livre de Krysin et Belikov, brillent par leur absence dans celui de Vaxtin et Golovko. Ces derniers reconnaissent la valeur des travaux de Karcevskij, Jakovlev, Šor, Seliščev, Polivanov, Jakubinskij, Larin, linguistes cités dans les deux ouvrages en question. Ces linguistes russes des années 1920-1930 ont porté un grand intérêt aux recherches de terrain, la méthode que Vaxtin et Golovko pensent indispensable pour une approche sociolinguistique du langage. C'est à mon avis pour cette raison que Baudouin de Courtenay ne figure pas dans le manuel de Vaxtin et Golovko, car ses considérations sur le rôle des facteurs sociaux ont été surtout d'ordre théorique ${ }^{6}$, on peut remarquer la même chose au sujet de Vinogradov. En ce qui concerne les auteurs russes cités, Vaxtin et Golovko mettent l'accent sur leurs travaux de terrain, somme toute, pas très nombreux et qui ne constituent un ensemble cohérent ni du point de vue théorique ni du point de vue méthodologique. Krysin et Belikov, quant à eux, mettent en avant les apports théoriques des mêmes linguistes, leurs «idées sans lesquelles la sociolinguistique actuelle ne pourrait pas exister", en reconnaissant "au nom de la justice, que la plus grande partie de ces travaux étaient spéculatifs et ne s'appuyaient pas sur des données langagières plus au moins conséquentes ${ }^{7}$.

Il y a des linguistes soviétiques que les deux manuels citent, mais pour des raisons différentes, voire opposées. C'est le cas de Šor et de Jakubinskij. Pour Krysin et Belikov, ces linguistes ont surtout contribué à une explication théorique de la différentiation linguistique, Šor avec son livre de 1926 Langue et société [Jazyk i obščestvo] et Jakubinskij

6. Krysin et Belikov retiennent surtout la thèse de Baudouin sur une différentiation horizontale (variation spatiale) et verticale (proprement sociale) de la langue. Il s'agit d'une élaboration théorique qui cherche à formuler quelques principes pour de futures recherches.

7. L.P. Krysin, V.I. Belikov, Sociolingvistika, p. 16. 
avec l'ouvrage de 1932 Essais linguistiques [Očerki po jazyku] (coécrit avec A.M. Ivanov) ${ }^{8}$. Pour Vaxtin et Golovko, le mérite de ces linguistes est d'avoir exposé devant un public russophone "des avancées occidentales dans le domaine de la sociologie du langage, avancées nouvelles pour cette époque " ${ }^{9}$. De ce point de vue, présentent un grand intérêt le livre de Šor cité plus haut et l'article de Jakubinskij «F. de Saussure sur l'impossibilité d'une politique linguistique» [«F. de Sossjur o nevozmožnosti jazykovoj politiki»] (1931).

Dans les années 1950, après la mise à mort officielle de la doctrine marriste, ont eu lieu des débats sur la voie à prendre pour la linguistique soviétique: fallait-il puiser dans les sciences du langage russes d'avant Marr, revenir en quelque sorte à la source? Ou était-il impératif de se tourner vers ce qu'il y avait de nouveau dans la linguistique étrangère (en occurrence vers le structuralisme américain), supposée être en avance comparée à la linguistique soviétique freinée par deux décennies de marrisme? Ou trouver une troisième voie? Les acteurs de cette polémique ne s'embarrassaient pas de nuances dans leur dénonciation de l'adversaire ${ }^{10}$. L'objectif du camp vainqueur, avec Vinogradov à sa tête, n'était pas d'éliminer le structuralisme en tant que principe théorique, mais seulement de l'enlever des mains des linguistes «bourgeois" pour en faire une véritable science (comprendre «russe»). Dans les sciences du langage en Union soviétique des années 1970-1980, le facteur social reçoit un statut ambigu, une ambiguïté qui n'a pas gêné à l'époque. Le rôle des facteurs sociaux dans le fonctionnement du langage y est, d'une part, hypertrophié, mais d'autre part, le social est constamment occulté au profit de facteurs purement systémiques, les investigations de terrain restant rares et, très probablement, pas bienvenues. Vaxtin et Golovko disent à ce propos:

A partir des années 1950, la linguistique soviétique sérieuse a évolué vers une étude structuraliste de la langue, avant tout dans les domaines de la phonologie, de la morphologie et de la syntaxe, et plus tard en

8. Dans les années 1930, Jakubinskij consacre également plusieurs articles à la même thématique dans les pages de la revue Literaturnaja učeba (cf. par exemple, L.P. Jakubinskij, «Klassovyj sostav sovremennogo russkogo jazyka. Jazyk krest'janstva", etc.)

9. N.B. Vaxtin, E.V. Golovko, Sociolingvistika i sociologija jazyka, p. 20.

Io. Cf. M. Schoenenberger, «La linguistique soviétique après N. Marr». 
sémantique et en typologie. Cette voie a apporté des résultats intéressants, cependant la composante sociale du problème n'avait pas été abordée, les rares publications sociolinguistiques se contentaient soit de résumer au choix quelques travaux occidentaux, soit de critiquer les théories bourgeoises, mais le plus souvent c'était les deux ${ }^{11}$.

Les auteurs qualifient une telle attitude de «réalisme socialiste ${ }^{12}$ en linguistique». La seule recherche de terrain d'envergure sur la différentiation sociale d'un usage précis a été l'ouvrage de Krysin (1974) dont Vaxtin et Golovko reconnaissent la valeur.

\section{Place des sources étrangères}

Aussi bien La sociolinguistique de Belikov et Krysin que La sociolinguistique et la sociologie du langage de Vaxtin et Golovko démontrent une connaissance approfondie de ce qui a été fait dans le domaine de la sociolinguistique dans le monde occidental. Les deux ouvrages citent et donnent parfois une analyse détaillée des notions sociolinguistiques de base chez leurs homologues anglophones comme H.A. Currie, D. Hymes, Ch. Ferguson, J. Gumperz, J. Fishman, W. Labov. Les deux ouvrages examinés donnent cependant une appréciation divergente de ces sociolinguistes: pour les auteurs de La sociolinguistique, la sociolinguistique anglophone n'est pas du tout pionnière dans le domaine et ne fait que poursuivre, sans le savoir parfois, le chemin ouvert par les Russes, les Tchèques et les Français dès la fin du XIX ${ }^{\mathrm{e}}$ siècle; pour Vaxtin et Golovko, en revanche, la sociolinguistique américaine

II. N.B. Vaxtin, E.V. Golovko, Sociolingvistika i sociologija jazyka, p. 23. Il y a eu quand même dans les années 1970 quelques articles qui soulignaient l'importance de la démarche sociolinguistique américaine et l'absence de travaux semblables en Union soviétique. Cf., par exemple, l'article de 1976 d'A.A. Leont'ev «Jazykovaja norma kak social'naja norma».

I2. Les auteurs font référence à la doctrine du «réalisme socialiste en littérature» imposée à tout œuvre littéraire soviétique dès les années 1930 qui proclamait une description, d'une part, "fidèle» à la réalité, mais qui devait en même temps véhiculer des valeurs de la société socialiste soviétique et poursuivait, par conséquent, des buts didactiques comme l'éducation par la littérature. Cette approche impose justement une déformation de la réalité pour des raisons idéologiques. $-M$. $S c h$. 
inaugure véritablement une étude sociale du langage dès les années 1950. Les auteurs présentent plusieurs autres ouvrages anglophones en la matière (entre autres, ceux de R.A. Hudson, R. Fasold, R. Trudgill, W.A. Foley). Pour Vaxtin et Golovko, les linguistes russes du premier tiers du $\mathrm{XX}^{\mathrm{e}}$ siècle avaient suivi en quelque sorte le mouvement général de l'intérêt envers les facteurs sociaux. Quant aux linguistes francophones d'orientation sociologisante de la même époque tels qu'A. Meillet, A. Sechehaye, Ch. Bally, ils sont absents dans le livre de Vaxtin et Golovko, qui ne s'étalent pas beaucoup sur les raisons de cette absence. "Question de goût", disent-ils sobrement ${ }^{13}$. A un autre endroit ${ }^{14}$, on peut lire que la sociolinguistique anglophone a été le résultat d'interactions et d'influences mutuelles entre la linguistique, la psychologie sociale et l'anthropologie de la culture, tandis que la sociolinguistique européenne (française en particulier) provenait d'interactions entre la linguistique, la sociologie et la philosophie. Serait-ce cette composante philosophique (jugée idéologique) qui aurait fait choisir à Vaxtin et Golovko une approche qui en est explicitement dépourvue? Je pense que c'est une des raisons de leur choix.

\section{Définition $d u$ «social»}

Vaxtin et Golovko refusent catégoriquement le parallélisme mécanique entre une langue, un territoire et une nation. Ils utilisent le terme de "communauté», "collectif», "groupe ethnique» pour parler du groupe d'individus dont les produits linguistiques sont l'objet d'une étude sociolinguistique. C'est un contexte extralinguistique qui influencerait l'emploi de certains moyens langagiers. Les éléments pertinents de ce contexte relèvent de la différenciation sociale de la communauté étudiée. Les auteurs ne font pas d'exposé détaillé de ce qu'ils entendent par «social», ils reconnaissent néanmoins une différenciation sociale hiérarchisée qui déterminerait certains emplois linguistiques. Pour plus de détails, Vaxtin et Golovko renvoient le lecteur aux travaux de sociologues, mais aussi à l'ouvrage du sociolinguiste américain W.A. Foley La linguistique anthropologique. Une introduction [Anthropological Linguistics: an Introduction]

I3. N.B. Vaxtin, E.V. Golovko, Sociolingvistika i sociologija jazyka, p. 6.

I4. Ibid., p. 13. 
de 1997 (où l'auteur consacre tout un chapitre à répertorier les facteurs sociaux à prendre en compte lors d'une investigation sociolinguistique) et à La sociolinguistique de Belikov et Krysin. Il est important de noter que Vaxtin et Golovko font une distinction entre le social et le linguistique (structure) dans le fonctionnement d'une langue: pour eux, la frontière entre un dialecte et une langue est conventionnelle et n'existe que $\mathrm{du}$ point de vue sociolinguistique, mais pas du point de vue purement linguistique (structurel). Ainsi, les auteurs prennent position contre une thèse connue du linguiste russe V.M. Žirmunskij (1891-1971) ${ }^{15}$ sur un lien causal mais déphasé dans le temps, entre la hiérarchisation d'une société (existence de classes) et l'existence de dialectes sociaux, thèse que Vaxtin et Golovko qualifient de "pseudomarxiste» ${ }^{16}$.

Krysin et Belikov renoncent au rapport direct entre les changements sociaux et linguistiques (thèse de L.P. Jakubinskij [1892-1945] et A.M. Ivanov sur l'isomorphisme entre la structure sociale et la structure linguistique) ${ }^{17}$. Cependant, la remise en question de cette thèse largement acceptée auparavant par la communauté des linguistes soviétiques est expliquée par une simple absence de recherches empiriques, elle n'est donc pas vraiment réfutée. Krysin et Belikov soutiennent par contre la thèse du retard de Žirmunskij, mentionnée plus haut, qui serait, d'après les auteurs, "partagée par la majorité des linguistes» ${ }^{18}$. Les auteurs citent Žirmunskij ${ }^{19}$ qui disait: "Pour décrire la structure d'une langue du point de vue de sa différenciation sociale, nous devons prendre en compte son passé et son avenir, c'est-à-dire toute la perspective potentielle de son évolution sociale» ${ }^{20}$. Pour Krysin et Belikov, comme apparemment pour beaucoup de linguistes russes, il n'y a pas de frontière entre le social et le linguistique, car l'évolution linguistique (structure de la langue) serait par définition conditionnée socialement et que, de surcroît, il est possible de la prévoir en raison de son retard sur les événements sociaux. Dans cette approche, l'objet de la linguistique a des contours flous, voire fluctuants. On peut même se demander si la linguistique a un objet propre.

I5. V.M. Žirmunskij, «Problema social'noj differenciacii jazykov», p. 32.

I6. N.B. Vaxtin, E.V. Golovko, Sociolingvistika i sociologija jazyka, p. 199, n. 1.

I7. L.P. Jakubinskij, A.M. Ivanov, Očerki po jazyku.

I8. L.P. Krysin, V.I. Belikov, Sociolingvistika, p. 92.

19. Ibid., p. 93.

2o. V.M. Žirmunskij, «Marksizm i social'naja lingvistika», p. 14. 
Les deux manuels reconnaissent une hiérarchisation sociale de toute communauté linguistique, y compris celle de la société russe. Les auteurs ne parlent pas explicitement d'une société de classes, mais la communauté russophone n'est plus présentée comme socialement homogène.

\section{Définition(s) de la sociolinguistique}

Dans le manuel de Krysin et Belikov, la sociolinguistique est définie comme un domaine de la linguistique se trouvant à la frontière entre la sociologie et la linguistique. Elle étudie l'action du milieu social sur la langue et sur le comportement langagier des locuteurs ${ }^{21}$; mais, mise à part l'étude de particularités langagières d'un individu en tant que représentant de diverses structures sociales, la sociolinguistique a pour tâche d'élucider le fonctionnement des langues dans la société ${ }^{22}$. Ainsi, il y a des phénomènes extralinguistiques qui influencent le comportement du locuteur et qui sont susceptibles d'intéresser le sociolinguiste, à savoir ceux propres aux communautés du type "groupe social» $[\text { socium }]^{23}$. Pour les auteurs, les groupes sociaux se caractérisent par une relative homogénéité et par une stabilité dans l'espace et dans le temps, ils possèdent une structure interne et sont liés entre eux pour former une société hiérarchisée. La notion de "groupe social» implique inévitablement la notion de "culture», dont les définitions sont multiples (plus de 300 dans le livre de Kluckhohn et Kroeber [1952] ${ }^{24}$, cité par Krysin et Belikov ${ }^{25}$ ), ce qui n'est pas gênant pour les auteurs, car "pour un linguiste une telle situation est banale et bien familière: des notions centrales pour la linguistique telles que "mot" ou "proposition" possèdent plusieurs dizaines de définitions» ${ }^{26}$. Krysin et Belikov ne reproduisent cependant qu'une seule définition de la culture, celle de l'ethnologue évolutionniste anglais du XIX ${ }^{\mathrm{e}}$ siècle E.B. Tylor, définition qui procède par énumération où la culture est "ce tout complexe comprenant

\footnotetext{
2I. L.P. Krysin, V.I. Belikov, Sociolingvistika, p. 16 sq.

22. Ibid., p. 173.

23. Ibid., p. 175.

24. C.K.M. Kluckhohn, A.L. Kroeber, Culture.

25. L.P. Krysin, V.I. Belikov, Sociolingvistika, p. 177.

26. Ibid.
} 
à la fois les sciences, les croyances, les arts, la morale, les lois, les coutumes et les autres facultés et habitudes acquises par l'homme dans l'état social» ${ }^{27}$. C'est une définition très large qui satisfait les auteurs, car pour eux, chaque groupe social possède une culture particulière et que ce qui définit le mieux une culture, c'est sa différence par rapport à une autre culture. L'appartenance à un groupe, et donc à une culture, se traduit nécessairement dans l'usage linguistique. La personne serait reconnue comme membre d'un groupe d'après sa façon de parler qui permettrait d'identifier l'appartenance sociale de l'individu.

L'«ethnie» (terme que les auteurs ne définissent pas spécialement) serait une communauté du type "groupe social» et par conséquent doit intéresser la sociolinguistique. Dans la perspective sociolinguistique en question, il se profile un complexe théorique où tout est lié: un groupe d'individus parlant la même langue, partageant la même culture et habitant le même territoire. C'est essentiellement au niveau lexical que se manifesteraient de manière flagrante des différences culturelles. Pour illustrer ces différences entre deux groupes sociaux ayant chacun sa propre langue (donc entre deux ethnies), Krysin et Belikov citent l'exemple des noms de "neige» chez les peuples du Grand Nord, exemple largement employé dans de nombreux ouvrages linguistiques et remis en cause par d'autres linguistes ${ }^{28}$.

Le livre de Vaxtin et Golovko ne donne pas une seule définition de la sociolinguistique, mais plusieurs, qui rejoignent dans les grandes lignes celle de Krysin et Belikov. A un moment, les auteurs incluent dans le champ des investigations sociolinguistiques l'influence des facteurs linguistiques sur la société, mais plus loin l'étude de cette influence incombe à une autre discipline scientifique, la sociologie du langage. Les auteurs citent, entre autres, la définition de la sociolinguistique

27. E.B. Tylor, La civilisation primitive, vol. I, p. 1 (les chercheurs russes se réfèrent à l'édition originale de ce livre datant de 1871, cf. L.P. Krysin, V.I. Belikov, Sociolingvistika, p. 177).

28. On trouve ce même exemple de l'existence de plusieurs termes pour dire "neige» chez les Esquimaux, entre autres, chez U. Eco dans Le signe. Histoire et analyse d'un concept, p. 152. Cet exemple a été l'objet d'une virulente critique dans le livre de G.K. Pullum, The Great Eskimo Vocabulary Hoax, and Other Irreverent Essays on the Study of Language, p. 162-171, où l'auteur démontre avec des arguments convaincants que l'esquimau ne possède pas plus de dénominations pour la neige que l'anglais. 
faite par Labov, considéré par beaucoup comme le fondateur de la sociolinguistique contemporaine:

[c'est] un domaine de connaissance qui étudie la structure de la langue et les changements linguistiques sur la base de données obtenues par l'investigation de l'usage quotidien de la langue ${ }^{29}$.

Ce qui importe pour les auteurs dans la définition de la sociolinguistique en tant que discipline à part entière, c'est sa méthode empirique inductive:

Le développement si fulgurant de la sociolinguistique durant ce dernier quart de siècle n'est pas dû à des spéculations de cabinet, mais aux découvertes empiriques faites parfois à partir d'un corpus exotique ${ }^{30}$.

Tout au long de leur ouvrage, Vaxtin et Golovko illustrent leurs propos par plusieurs exemples tirés essentiellement de travaux sociolinguistiques anglo-américains. Par ces illustrations, les auteurs tiennent à démontrer la primeauté des recherches de terrain pour la constitution même des concepts de base en sociolinguistique. Vaxtin et Golovko redonnent en quelque sorte ses lettres de noblesse à une linguistique active qui apprend en se confrontant au terrain, et non pas forcément en fonction de méthodes et d'intentions prédéterminées. Les exemples russes sont peu nombreux, vu la rareté des travaux de terrain en Russie; pour les auteurs, «tout est encore à faire». Une affirmation qui tranche franchement avec l'idée de la suprématie de la linguistique sociale russe, défendue dans le manuel de Krysin et Belikov. Dans ce dernier ouvrage, riche en exemples en russe, ces derniers sont majoritairement tirés d'œuvres littéraires et cinématographiques russes de différentes époques. D’ailleurs, les toutes premières lignes du premier chapitre "Qu'étudie la sociolinguistique?» ["Čto izučaet sociolingvistika?»] du manuel de Krysin et Belikov présentent une citation tirée d'une œuvre "immortelle» ${ }^{31}$ de l'écrivain soviétique K.I. Čukovskij. Dès une première lecture rapide des deux manuels examinés, c'est le choix des exemples qui attire l'attention et qui est révélateur de deux approches divergentes de la sociolinguistique.

29. N.B. Vaxtin, E.V. Golovko, Sociolingvistika i sociologija jazyka, p. 16.

30. Ibid., p. 26.

3I. L.P. Krysin, V.I. Belikov, Sociolingvistika, p. 9. 
Pour Vaxtin et Golovko, les notions comme "nation ", "peuple», "ethnie» ne sont importantes que si elles sont présentes dans l'imaginaire du sujet parlant et qu'elles influencent son comportement langagier. Les auteurs s'attardent néanmoins sur les défnitions de ces notions formulées par divers chercheurs. Ainsi, pour la notion d'"ethnie", employée mais pas vraiment définie dans le manuel de Krysin et Belikov, Vaxtin et Golovko présentent les points de vue primordialiste [primordial ethnicity] d'E. Shils, constructiviste (B. Anderson) et situationniste (F. Barthes). Vaxtin et Golovko présentent plus en détail la conception de Barthes dans laquelle l'ethnie est quelque chose de construit aussi bien de l'intérieur (sentiment des sujets d'appartenir à la même ethnie; de ce point de vue il s'agit d'un groupe) que de l'extérieur (sentiment d'être reconnu en tant que groupe ethnique par l'entourage). Les auteurs de La sociolinguistique et la sociologie du langage refutent la conception de l'ethnie comme une caractéristique innée, fondamentale, inconditionnelle et constante d'un individu. Les frontières d'un groupe ethnique et d'un groupe linguistique ne coïncideraient pas, le sentiment "ethnique" n'étant qu'une des dimensions d'un concept plus large qui est l'identité sociale.

\section{Notion de "norme linguistique»}

Dans le manuel de Vaxtin et Golovko, c'est une grande absente, en tout cas en tant que notion linguistique, mais on y trouve la notion de "choix entre les variantes linguistiques conditionnées socialement». Pour les auteurs, le sociolinguiste doit observer et noter les variantes. Il les relève, cherche à les expliquer quand c'est possible (en faisant intervenir, par exemple, les notions de "stéréotype", de "préjugé culturel», de "prestige», etc.), mais ne juge pas et ne fait pas de prédictions.

Dans l'ouvrage de Krysin et Belikov, la notion de «norme linguistique» est une des notions centrales. Elle est étroitement liée à celles de "choix", de "système (structure) linguistique» et de "langue littéraire»:

La norme est un ensemble de règles gouvernant le choix et l'usage des moyens linguistiques. La notion de norme est indissolublement liée 
à celle de langue «littéraire» qu’on appelle d'ailleurs fréquemment normée ${ }^{32}$.

Si, pour Vaxtin et Golovko, la norme linguistique est purement structurelle, décrite dans le cadre de la linguistique structurale et précède une recherche sociolinguistique, mais n'est pas l'objet de cette dernière, pour Krysin et Belikov la norme est au centre de toute investigation sociolinguistique, elle est à la fois le point de départ, l'objet de l'étude et le critère de l'évaluation des produits linguistiques:

La norme remplit une fonction sociale et culturelle de haute importance. Tous les domaines socialement importants sont desservis par la langue normée... La norme joue le rôle d'un filtre: elle laisse passer dans l'usage littéraire tout ce qu'il y a de marquant, de juste, de savoureux [jarkoe, metkoe, sočnoe] dans l'usage du peuple (des gens) [ $v$ narodnoj reči $]$ et fait barrière, élimine tout ce qui est fortuit, terne, inexpressif. En même temps, la norme est dynamique: elle ne divise pas d'une façon rigide les moyens linguistiques en bons et mauvais, elle ne prescrit pas d'employer toujours les premiers et jamais les seconds. On appelle "orientation communicative vers un but précis» [kommunikativnaja celesoobraznost'] la dépendance de la norme des conditions dans lesquelles se réalise la parole ${ }^{33}$.

On retrouve le même raisonnement circulaire entre la norme, le système et les facteurs sociaux déjà dans les travaux de Krysin des années 1970-1980 ${ }^{34}$ - la norme est considérée tantôt comme sociale, relevant de facteurs externes au système, elle est alors plus restrictive que le système:

L'antinomie système/norme consiste en ce que le système "permet" tout ce qui ne contredit pas les lois de la langue donnée, tandis que la norme trie, filtre ce qui est permis par le système, mais ne laisse pas passer jusqu'à l'usage tout ce que "permettent» les possibilités systémiques de la langue ${ }^{35}$,

32. Ibid., p. 39.

33. Ibid., p. 40.

34. L.P. Krysin, Russkij jazyk po dannym massovogo obsledovanija; id., Sociolingvističeskie aspekty izučenija sovremennogo russkogo jazyka.

35. L.P. Krysin, V.I. Belikov, Sociolingvistika, p. 106. 
tantôt la norme sociale (facteurs sociaux qui font choisir au sujet une forme au détriment d'une autre) fait partie de la structure linguistique elle-même:

Sur d'autres tronçons du système linguistique cette influence [des facteurs sociaux] prend des formes plus cachées et plus complexes. Il s'avère, par exemple, que les facteurs sociaux ne font pas que créer les conditions dans lesquelles évolue et fonctionne la langue, mais qu'ils sont des composants de la structure des unités linguistiques. Au prime abord, cela semble peu probable. Mais les faits en témoignent: le social pénètre dans le tissu de la langue et ne fait pas que servir d'entourage pour son emploi ${ }^{36}$.

Dans cette vision de la sociolinguistique, les normes sociales relevant de la structure linguistique peuvent être connues autrement que par la voie des recherches de terrain. Comparée à l'approche de Vaxtin et Golovko, celle de Krysin et Belikov marque une séparation importante entre les sociolinguistes et leur objet d'étude, les usages linguistiques.

\section{Langue «littéraire» (ou son équivalent)}

Les raisons des divergences entre deux manuels de sociolinguistique deviennent beaucoup plus claires si l'on prend en considération l'évolution des idées et des concepts en linguistique soviétique et postsoviétique durant les décennies précédentes. La théorie dominante de cette période est celle des langues dites "littéraires». Krysin a été une des figures centrales des recherches menées dans le cadre de cette théorie en Union soviétique. Dans La sociolinguistique de Krysin et Belikov, on retrouve pratiquement les mêmes postulats et définitions. La langue «littéraire» $y$ est définie comme

[...] une variante de la langue nationale la plus appropriée à la communication dans le plus grand nombre de domaines sociaux, à savoir dans la science, dans l'enseignement, dans le monde diplomatique et dans le droit, dans les rapports de travail entre les gens et les institutions, dans la communication quotidienne des gens cultivés ${ }^{37}$.

36. Ibid., p. 163.

37. Ibid., p. 42. 
La langue «littéraire» est avant tout un système possédant des normes stables et obligatoires, polyfonctionnel (en usage dans plusieurs domaines d'emploi). Le point qui est thématisé davantage dans l'ouvrage de 2001, c'est le prestige social de la langue «littéraire». En effet, le prestige de l'usage "littéraire» a été fréquemment mentionné dans les travaux soviétiques des années 1970-1980, tout en étant occulté dans les conclusions des linguistes au profit des propriétés purement systémiques, intrinsèques de la langue «littéraire». Krysin et Belikov distinguent la langue «littéraire» en tant que construction théorique unifiée et normative, d'une part, et en tant que réalité, "un système communicatif réel", d'autre part, qui fonctionnerait dans des "conditions nationales concrètes " ${ }^{38}$. Ainsi, la variabilité de moyens d'expression "littéraires" (existence de formes «littéraires» considérées comme synonymiques) ne relèverait plus exclusivement de processus opérant dans la structure «littéraire» et reflétant une "compétition» entre une forme "progressiste», ayant de l'avenir, et une autre condamnée à disparaître, mais également de facteurs sociaux tels que l'âge, la profession, le niveau d'instruction, etc. Krysin et Belikov reconnaissent l'existence de variantes régionales ou substrats de la langue «littéraire». La variation linguistique due au fonctionnement de la langue dans l'espace trouve ainsi un statut théorique. Rappelons que dans les décennies précédentes, il y a eu des propositions de reconnaître les variantes régionales de la langue "littéraire", c'était l'avis de V.N. Jarceva ${ }^{39}$ et de quelques autres linguistes essentiellement de Léningrad. Ces propositions ont été critiquées et rejetées, la langue «littéraire» russe avait gardé dans les écrits des linguistes soviétiques son unicité pour tout le territoire russophone. Dorénavant, le manuel de sociolinguistique de Krysin et Belikov admet, au moins au niveau de la réalisation linguistique, l'existence de variation «littéraire» régionale et cite, entre autres, le travail de Jarceva de 1977 qui postulait déjà une stratification régionale de la langue «littéraire». Les auteurs restent néanmoins fidèles au postulat de la théorie des langues "littéraires" sur le progrès de la langue «littéraire». Cette dernière atteindrait avec le temps un haut niveau de développement et changerait moins rapidement, voire se figerait. Ainsi, la position de Krysin dans les années 2000 n'est guère

38. Ibid., p. 99.

39. V.N. Jarceva, «Sootnositel'nost' regional'nyx i social'nyx variantov jazyka v plane stilja i normy». 
différente de sa position dans les années 1970-1980 : c'est la structure de la langue «littéraire" préexistant à l'investigation qui possèderait des propriétés qui rendraient cette langue plus viable que tous les autres usages et qui expliquerait son expansion et sa fixation.

Mis à part la variation «littéraire» régionale qui est dorénavant acceptée, mais en même temps relativisée, toute variation dans la langue «littéraire» elle-même ne peut être que fonctionnelle: des moyens d'expression synonymiques appartiendraient forcément à des styles fonctionnels différents, c'est-à-dire n'apparaîtraient pas dans un même domaine d'emploi. La paternité du concept de style fonctionnel est attribuée traditionnellement à l'Ecole de Prague. En Union soviétique, c'est la classification des styles fonctionnels de D.N. Šmelev ${ }^{40}$ qui servait de référence en la matière. Dans l'ouvrage de Krysin et Belikov, la notion de «styles fonctionnels» est centrale pour parler d'une stratification de la langue «littéraire». On assiste cependant à un changement important par rapport aux travaux des décennies précédentes. Premièrement, les auteurs relèvent une non-correspondance possible, voire systématique entre la situation de communication et le style choisi par le locuteur. Ce serait un des centres d'intérêt de la sociolinguistique. Deuxièmement, les auteurs ne considèrent plus les styles fonctionnels en tant que sous-systèmes de la langue tels que les concevait Šmelev. Les auteurs de La sociolinguistique associent désormais les styles fonctionnels aux "genres de la parole», notion présente dans la linguistique russe et la sociolinguistique anglo-américaine depuis longtemps. Krysin et Belikov citent le travail du linguiste pétersbourgeois K.A. Dolinin (1978) en tant qu'exemple d'une description de styles de la parole et mentionnent Labov ${ }^{41}$. Il est important de noter que, dans cette acceptation, les styles fonctionnels relèvent de la parole et non plus de la langue en tant que structure. On ne trouvera pas dans l'ouvrage de Krysin et Belikov une explication plus détaillée de ce revirement. Le fait que les «styles fonctionnels» apparaissent comme concept à contenus variables ne gêne pas les auteurs. Il leur semble important de souligner une continuité et même une filiation

40. D.N. Šmelev, Russkij jazyk v ego funkcional'nyx raznovidnostjax.

4I. Les auteurs ne citent pas M.M. Baxtin (Bakhtine), qui a été pourtant parmi les premiers à employer la notion de "genres de la parole" [rečevye žanry] sur le sol russe dans son article "Problema rečevyx žanrov" écrit en 1953 mais paru beaucoup plus tard, en 1978. 
entre les travaux soviétiques sur la langue «littéraire» et la sociolinguistique actuelle. D'ailleurs, en guise de conclusion, Krysin et Belikov citent Vinogradov, considéré comme le fondateur de la théorie des langues "littéraires", en tant que pionnier de l'analyse sociolinguistique qui a pressenti les problèmes centraux de la sociolinguistique à venir, a été «à la source» de cette discipline et a donné «un exemple brillant d'une analyse sociolinguistique» surtout dans ses travaux sur la stylistique ${ }^{42}$, dans lesquels justement les styles fonctionnels étaient conçus comme des sous-systèmes de la langue.

Une autre problématique sociolinguistique «héritée» de la théorie des langues «littéraires» et de la "culture de la langue» (en tant que discipline linguistique) est l'étude des usages «non littéraires». Parmi les usages "non littéraires», on retrouve les dialectes, le prostorečie (forme orale non codifiée et non littéraire), les jargons. La clé de cette différence réside, pour Krysin et Belikov, dans la différence de leurs normes respectives:

La différence entre la norme "littéraire» et celle d'un dialecte ou d'un jargon réside dans le fait que la norme «littéraire» est cultivée consciemment: elle est fixée dans les dictionnaires et les grammaires, elle est enseignée à l'école, elle est l'objet d'une propagande dans les livres, les émissions radio et télé, toute communication cultivée entre les gens se fait d'habitude conformément aux normes de la langue «littéraire». Dans les dialectes, et encore moins dans le prostorečie et les jargons, ce n'est pas le cas: il y existe une tradition dans l'usage des moyens linguistiques, mais aucun locuteur du dialecte en question ne le préserve d'influences externes, personne n'y cultive de façon consciente et systématique des étalons de communication dialectale ${ }^{43}$.

Autrement dit, la différence entre les normes «littéraires» et les autres relève de la codification des premières et de l'absence de codification pour les secondes, qui sont également appelées «formes non codifiées» ${ }^{44}$. Krysin et Belikov leur attribuent plusieurs traits communs tels que l'absence de normes conscientes, mais plutôt une tradition que les auteurs conçoivent comme inconsciente. La question à laquelle les auteurs

42. L.P. Krysin, V.I. Belikov, Sociolingvistika, p. 98.

43. Ibid., p. 41.

44. Ibid., p. 98. 
ne répondent pas est celle de savoir si la norme et la tradition ont un rapport uniquement à la codification ou ont quelque chose à voir avec les propriétés systémiques spécifiques de la norme «littéraire». En effet, d'une part, le manuel de Krysin et Belikov thématise et met en relief des facteurs sociaux comme "prestige", "propagande", "culture» dans le fonctionnement du langage et insiste sur la nécessité d'étudier une communication concrète ancrée dans le social. De ce point de vue, le manuel en question tranche avec les écrits classiques sur la langue «littéraire" des décennies antérieures, y compris avec les travaux de Krysin lui-même. D'autre part, Krysin et Belikov maintiennent le postulat sur l'existence des normes "littéraires" spécifiques par leur nature dans le système même de la langue...

Pour Golovko et Vaxtin, le problème de la langue «littéraire» n'en est pas un. La langue « littéraire» que les auteurs appellent également «standard» est un usage reconnu, en tant que le plus prestigieux, comme une «langue» et non comme un "dialecte», la reconnaissance et le prestige étant le résultat d'un acte politique ${ }^{45}$, de la codification et de la diffusion massive de cet usage. On ne trouvera pas dans ce manuel-là de mention d'une structure linguistique particulière pour la langue "littéraire» ou standard. C'est une définition purement sociologique (prestige, reconnaissance, diffusion massive). La "vitalité» d'une langue en usage dépendrait de facteurs externes à la langue: de son statut dans la communauté linguistique, de la situation démographique, du soutien institutionnel. C'est un point de vue communément admis dans la sociolinguistique actuelle.

Comme Krysin et Belikov, Vaxtin et Golovko se préoccupent eux aussi du problème du choix de moyens linguistiques particuliers en fonction de la situation de communication. Les auteurs de La sociolinguistique et la sociologie du langage emploient le terme de "registres" qu'ils empruntent à M.A.K. Halliday ainsi que sa classification de registres linguistiques ${ }^{46}$. Les registres en question relèvent de la parole et non pas

45. Les auteurs citent la boutade devenue classique d'U. Weinreich dans son livre Languages in Contact (1953) pour définir une langue: «Une langue, c'est un dialecte qui dispose d'une armée» (p. 43).

46. M.A.K. Halliday, Language as Social Semiotic: the Social Interpretation of Language and Meaning. 
de la langue. Vaxtin et Golovko ne font aucune référence au concept de «styles fonctionnels» qu'ils doivent certainement connaître.

\section{Conclusion}

Rappelons que La sociolinguistique de Krysin et Belikov et La sociolinguistique et sociologie du langage de Vaxtin et Golovko sont les seuls manuels de sociolinguistique parus en Russie depuis la fin des années 1970. C'est avant tout par l'intermédiaire de ces ouvrages que les étudiants russes en linguistique appréhendent l'étude sociale du langage. Il faut savoir que les programmes de cours universitaires sont définis dans les grandes lignes par le Ministère de l'éducation nationale et que la sociolinguistique en fait partie ${ }^{47}$. Le manuel de Vaxtin et Golovko est le fruit de plusieurs années de cours de sociolinguistique donnés par les auteurs à l'Université Européenne de Saint-Pétersbourg aux jeunes doctorants en sciences du langage et peut être utilisé en tant qu'ouvrage de référence par les enseignants. Le manuel de Krysin et Belikov est signé par des linguistes réputés en Russie pour leurs apports dans l'étude des facteurs sociaux dans le fonctionnement du langage et c'est ce manuel-là qui est recommandé à l'enseignement supérieur par le Conseil scientifique et didactique de la direction du Ministère de l'éducation supérieure $[N($ aučno $) M$ (etodičeskij)S(ovet) po filologii U(čebno-)M(etodičeskogo) $O$ (b"edinenija universitetov R(ossijskoj)F(ederacii)]. C'est par conséquent le livre de Krysin et Belikov qui est actuellement un ouvrage de référence obligatoire pour toutes les facultés de lettres en Russie. Cependant, et c'est la conclusion à laquelle je suis arrivée au terme de mon analyse, le manuel en question n'est pas à proprement parler un manuel de sociolinguistique. Certes, il présente un large éventail d'auteurs et de méthodes sociolinguistiques contemporains, mais il reste ancré dans une tradition scientifique et méthodologique spécifiquement russe des époques précédentes. La sociolinguistique de Krysin et Belikov réaffirme les postulats essentiels de la théorie soviétique des langues "littéraires" sur l'existence, l'expansion et les propriétés systémiques à part d'une structure linguistique appelée «littéraire». Ces postulats continuent de gouverner

47. Cf. Gosudarstvennyj obrazovatel'nyj standart vysšego professional'nogo obrazovanija du 10 mars 2000. 
la recherche linguistique en Russie. L'apport de la sociolinguistique anglo-américaine y est reconnu, mais relativisé au profit de sources scientifiques russes. La sociolinguistique russe telle qu'elle apparaît dans le manuel de Krysin et Belikov est une discipline renfermée sur elle-même, il semble plus important pour les auteurs de préserver une continuation avec les théories linguistiques élaborées sur le sol russe antérieurement que de confronter ces théories à des pratiques langagières réelles.

Par contre, le manuel de Vaxtin et Golovko s'inscrit entièrement dans une lignée d'écrits sociolinguistiques occidentaux, il rompt de ce fait avec l'héritage soviétique mais ne thématise pas cette rupture.

Margarita SCHOENEnBerger

Université de Lausanne 


\section{BIBLIOGRAPHIE}

Baxtin, Mixail Mixajlovič (Bakhtine, Mikhail), «Problema rečevyx žanrov", Literaturnaja učeba, 1 (1978), p. 200-219.

Dolinin, Konstantin Arkad'evič, Stilistika francuzskogo jazyka, Leningrad, Prosveščenie, 1978.

Eco, Umberto, Le signe. Histoire et analyse d'un concept [Segno], adapté de l'italien par Jean-Marie Klinkenberg, Paris, Le livre de poche, 1992 (première édition parue à Bruxelles, Editions Labor, 1988).

Ferguson, Charles Albert, "Diglossia», Word, 15 (1959), p. 325-340.

Foley, William A., Anthropological Linguistics: an Introduction, Oxford, Blackwell, 1997.

Gumperz, John J., "The Speech Community", in International Encyclopedia of the Social Sciences, eds David L. Sills, Robert K. Merton, New York, Macmillan Free Press, 1968, p. 381-386.

Halliday, Michael Alexander Kirkwood, Language as Social Semiotics: the Social Interpretation of Language and Meaning, London, Arnold, 1978.

Jakubinskij, Lev Petrovič, "Klassovyj sostav sovremennogo russkogo jazyka. Jazyk krest' janstva», Literaturnaja učeba, 4 (1930), p. $80-92$.

—, «F. de Sossjur o nevozmožnosti jazykovoj politiki», Jazykovedenie i materializm, 2 (1931), p. 91-104.

Jakubinskij, Lev Petrovič, Ivanov, An. M., Očerki po jazyku, Moskva/ Leningrad, GIXL, 1932.

Jarceva, Viktorija Nikolaevna, "Sootnositel'nost' regional'nyx i social'nyx variantov jazyka v plane stilja i normy", in Social'naja i funkcional'naja differenciacija literaturnyx jazykov, otv.red./éds V.N. Jarceva, Mirra Moiseevna Guxman, Moskva, Nauka, 1977, p. 12-26.

Kluckhohn, Clyde Kay Maben, Kroeber, Alfred L., Culture: a Critical Review of Concepts and Definitions, Cambridge, Peabody Museum of American Archeology and Ethnology, Harvard University, 1952. 
Krysin, Leonid Petrovič, Russkij jazyk po dannym massovogo obsledovanija, Moskva, Nauka, 1974.

-, Sociolingvističeskie aspekty izučenija sovremennogo russkogo jazyka, Moskva, Nauka, 1989.

Krysin, Leonid Petrovič, Belikov, Vladimir Ivanovič, Sociolingvistika, Moskva, Izdatel'stvo Rossijskogo gosudarstvennogo gumanitarnogo universiteta, 2001.

LeOnt'Ev, Aleksej Alekseevič, "Jazykovaja norma kak social’naja norma", in otv.red./éd. Venori Mixajlovič Kvačaxija, Problemy social'noj psixologii, Tbilisi, Izdatel'svo Tbilisskogo universiteta, 1976, p. 93-97.

Pullum, Geoffrey K., The Great Eskimo Vocabulary Hoax, and Other Irreverent Essays on the Study of Language, Chicago, University of Chicago Press, 1991.

Schoenenberger, Margarita, "Une sociolinguistique prescriptive: la théorie des langues "littéraires" dans la linguistique soviétique des années 60-90", Langage et société, 110 (2004), p. 25-52.

-, "La linguistique soviétique après N. Marr: linguistes, structuralisme et "révolution scientifique et technologique" ", Cahiers de l'ILSL, 26 (2009), p. 159-174.

ŠMELEv, Dmitrij Nikolaevič, Russkij jazykv ego funkcional'nyx raznovidnostjax, Moskva, Nauka, 1978.

Šor, Rozalija Osipovna, Jazyk i obščestvo, Moskva, Rabotnik prosveščenija, 1926.

Švejcer, Aleksandr Davidovič, Nikol'skij, Leonid Borisovič, Vvedenie v sociolingvistiku, Moskva, Vysšaja škola, 1926.

Tylor, Edward Burnett, La civilisation primitive [Primitive Culture], tr. de l'anglais par Pauline Brunet sur la deuxième édition, Paris, Reinwald, 1876-1878, vol. 1-2.

VAXтın, Nikolaj Borisovič, Golovко, Evgenij Vasil'evič, Sociolingvistika i sociologija jazyka, Sankt-Peterburg, Evropejskij centr v SanktPeterburge, 2004.

Weinreich, Uriel, Languages in Contact, New York, Publications of the Linguistic Circle of New York, 1953.

ŽIrmunskiJ, Viktor Maksimovič, «K probleme social'noj differenciacii jazykov", in otv.red./éd. Fedot Petrovič Filin, Jazyk i obščestvo, Moskva, Nauka, 1968, p. 22-39. 
—, "Marksizm i social'naja lingvistika», in Voprosy social'noj lingvistiki, otv.red./éds Agnija Vasil'evna Desnickaja, V.M. Žirmunskij, Ljudmila Stepanovna Kovtun, Leningrad, Nauka, 1969, p. 5-25. 
